\title{
La alergología en el sistema sanitario público de la Comunidad de Madrid
}

\section{Hernández Fernández de Rojas ${ }^{1}$, J. Sastre Domínguez ${ }^{2}$}

\section{Sr. Director:}

Hemos leído con gran interés el artículo original sobre la situación actual de la especialidad de alergología en el sistema sanitario público de la Comunidad de Madrid $(\mathrm{CM})^{1}$. Como señalan los autores, se trata de los primeros datos publicados sobre la organización de la alergología en España.

La Sociedad Española de Alergología e Inmunología Clínica (SEAIC), que aglutina a más de 860 socios numerarios, especialistas de alergología repartidos por todo el país, no puede ser ajena a esta iniciativa de la CM, pionera en el análisis y planificación estratégica de la atención alergológica. Como sociedad profesional y científica la misión de la SEAIC está orientada a mejorar la atención de los pacientes con enfermedades alérgicas, fomentando la práctica clínica de excelencia, centrada en las necesidades del paciente y de la población y marcando unos estándares a nivel del territorio nacional ${ }^{2}$.

Los datos sobre la atención alergológica en el Servicio Madrileño de Salud (SERMAS) confirman que la Comunidad de Madrid (CM) cuenta con una amplia red de profesionales y servicios que probable- mente excede a la de otras muchas comunidades. Los autores, además de analizar las actividades asistenciales, docentes e investigadoras, han realizado un análisis estratégico detectando áreas de mejora, que se podrían hacer extensibles a todas las comunidades. En concreto, proponen optimizar la organización asistencial aprovechando la cercanía física entre los diferentes centros y las nuevas tecnologías, mejorando así la eficiencia. También señalan la necesidad de mejorar la codificación de las enfermedades alérgicas (CIE 9 y CIE-10 resultan poco útiles), crear herramientas para el registro uniforme de la actividad, que refleje la complejidad de los procedimientos propios de la especialidad, tanto diagnósticos como terapéuticos. Así mismo, proponen potenciar la docencia de la alergología en las universidades, fomentando el acceso de los médicos alergólogos a los departamentos de medicina.

Pero sin lugar a dudas, la información más relevante del trabajo es la relativa al volumen y características de la actividad asistencial. El trabajo de recogida de información fue realizado en 2011, sobre datos asistenciales de 2010. En total colaboraron 117 especialistas en alergología de 24 hospi-
1. Presidenta del Comité de Asuntos Profesionales de la Sociedad Española de Alergología e Inmunología Clínica.

2. Presidente de la Sociedad Española de Alergología e Inmunología Clínica.

\author{
Correspondencia \\ Joaquín Sastre Domínguez \\ Fundación Jiménez Díaz \\ Avd. Reyes Católicos, 2 \\ 28040 Madrid \\ jsastre@sjd.es
}


tales públicos de la $\mathrm{CM}$, con una población estimada de 6,5 millones de habitantes. Durante el periodo analizado se realizaron 96.775 primeras visitas, procediendo el 84\% de atención primaria (AP). Estos datos suponen una tasa de nuevas consultas relativamente baja $(1,49 \%)$, para la elevada prevalencia de la patología alérgica (máxima para la rinitis, que afecta al $22 \%$ de la población) y plantean la cuestión sobre si la accesibilidad a la atención alergológica es la adecuada. Sobre este punto se puede argumentar que, aunque muy prevalente, la enfermedad alérgica causa escasa morbilidad y con adecuada formación, podría ser controlada de forma sintomática en $\mathrm{AP}$, una vez establecido el adecuado diagnóstico alergológico. Además, las manifestaciones alérgicas más graves, son resueltas por los servicios de emergencia, desde no siempre se remiten los pacientes a alergología con el fin de identificar los desencadenantes de los episodios alérgicos y así poder prevenir su recurrencia. Estas cuestiones son extensibles a todas las comunidades.

En cuanto a la actividad asistencial en los 24 centros analizados, llama la atención que, aunque la alergología es una especialidad de ámbito fundamentalmente ambulatorio, la mayor parte de los servicios (18 de los 24) se encuentran en centros sanitarios de II y III nivel, contando con una media de 4,6 profesionales. Esta situación también es común en el resto del país y está en relación con el modelo sanitario que ha impulsado la asistencia especializada desde los grandes centros sanitarios. Sin embargo, siendo AP el principal proveedor de pacientes y la mayoría de las enfermedades alérgicas de baja morbilidad, se podrían sugerir otros escenarios más eficientes para la atención alergológica, siguiendo las indicaciones de Ley de Cohesión y Calidad del Servicio Nacional de Salud ${ }^{3}$. En este sentido, la propuesta de implantar consultas de alergología en centros de especialidades ambulatorios, parece más razonable y eficiente. Con el fin de no restar oportunidades, tanto a los pacientes en cuanto al acceso a procedimientos diagnósticos y/o terapéuticos, como a los profesionales, estos centros se podrían vincular a los cen- tros de II o III nivel. Este modelo, ya existe en algunos distritos sanitarios del país, pero no ha sido suficientemente analizado ni explotado.

El trabajo incluye una original aportación: la clasificación de las prestaciones complementarias en alergología dependiendo de su complejidad y analiza la implantación de las prestaciones más complejas en los diferentes centros. Esta clasificación resulta razonable, pero podría complementarse con criterios de idoneidad, puesto que, aunque sean de poca complejidad y riesgo, no en todos los centros deberían realizarse todo tipo de prestaciones (olfatometría, endoscopia nasal, esputo inducido). En este contexto, se deberían establecer unas recomendaciones generales sobre la dotación y prestaciones para cada nivel de la asistencia alergológica.

Llama la atención en el trabajo de Domínguez-Ortega, las escasas alusiones a los tratamientos con inmunoterapia específica con alérgenos, modalidad terapéutica exclusiva de la especialidad. En la tabla 4, que incluye el número de exploraciones y procedimientos terapéuticos realizados durante 2010 , se recoge la información sobre la administración de inmunoterapia con inhalantes (la más frecuente) e himenópteros, con una media de 1.667,25 tratamientos por servicio. Históricamente estos tratamientos se han administrado en el entorno de AP. Sin embargo, en los últimos años se han organizado en torno a los servicios de alergología unidades de inmunoterapia, con el fin de controlar y asegurar su correcta administración. La SEAIC ha revisado recientemente la dotación, prestaciones y ámbitos de actuación de estas unidades.

En cuanto a las actividades docentes, llama la atención el elevado porcentaje de especialistas con el grado de doctor (41\%), en posible relación con el nivel asistencial y la vinculación con las universidades de los centros sanitarios analizados. Sin embargo, solo 5 profesionales $(4,27 \%)$ realizaban actividades docentes como profesores asociados de universidad. Esta carencia es también general en todas las comunidades y debería proponerse como 
un objetivo estratégico en la especialidad de alergología.

El tamaño y la dotación de los servicios de alergología de la CM destacan también por su elevada capacidad de formación especializada, con 10 centros acreditados y 2,78 plazas de MIR por centro. Aunque se trata de centros con muchos años de experiencia y tradición docente, el número de especialistas en formación excede con mucho las necesidades de reposición de especialistas para la CM y supone un detrimento en la convocatoria de plazas para el resto de las comunidades.

La actividad investigadora es relevante y cubre la investigación clínica, participación en proyectos de investigación, publicaciones, ponencias y comunicaciones a congresos nacionales e internacionales, pero no es homogénea y no depende del nivel asistencial. Este aspecto se repite también en el resto del país y está en relación con el bajo nivel de exigencia y reconocimiento de la investigación en el entorno sanitario, incluso en hospitales de III nivel, a pesar de las recomendaciones recogidas en la Ley de Ordenación de Profesiones Sanitarias ${ }^{4}$.

En conclusión, el trabajo referido constituye un importante documento que sin duda servirá como punto de partida para el análisis general de la atención alergoló- gica, centrada en las necesidades del paciente y de la población, con el objetivo de abordar su planificación estratégica. Para ello es imprescindible conocer el sustrato científico de la alergología, estimar el incremento previsible de la prevalencia de las enfermedades alérgicas, analizar la organización de los recursos disponibles y evaluar los procedimientos diagnósticos y terapéuticos de la práctica clínica alergológica. La SEAIC, por su grado de implantación, compromiso, capacidad y vocación, debe liderar la planificación estratégica de la alergología y actuar como interlocutor y motor de dicha planificación.

\section{BIBLIOGRAFÍA}

1. Domínguez-Ortega J, Ruiz Hornillos J, Chivato T, de Lahoz B, Elices A, Iglesias A, Quirce S, Vega F, Rodríguez Mosquera M. La alergología en el sistema sanitario público de la Comunidad de Madrid: situación actual y oportunidades en el sistema sanitario público. An Sist Sanit Navar 2014; 37: 317-327.

2. http://www.seaic.org/inicio/

3. Ley $16 / 2003$, de 28 de mayo, de Cohesión y Calidad del Sistema Nacional de Salud. BOEA-2003-10715

4. Ley $44 / 2003$, de 21 de noviembre, de Ordenación de las Profesiones Sanitarias. BOEA-2003-21340 